\title{
Molecular Dynamics Investigation of the Influence of the Hydrogen Bond Networks in Ethanol/Water Mixtures on Dielectric Spectra
}

\author{
Javier Cardona, ${ }^{*}+\odot$ Martin B. Sweatman, ${ }^{*}$ and Leo Lue \\ ${ }^{\dagger}$ Department of Chemical and Process Engineering, University of Strathclyde, James Weir Building, 75 Montrose Street, Glasgow G1 \\ 1XJ, United Kingdom \\ ${ }^{\ddagger}$ School of Engineering, The University of Edinburgh, The King’s Buildings, Sanderson Building, Mayfield Road, Edinburgh EH9 3JL, \\ United Kingdom
}

Supporting Information

ABSTRACT: The dielectric response of fluids to electromagnetic radiation in the microwave region originates from processes occurring at the molecular level. Understanding these processes in more detail is relevant to many fields, such as microwave heating, fluid mixing, and separation technologies. In this work, we use molecular dynamics (MD) simulations to study the dielectric spectra of ethanol/water mixtures. We compare our predictions with experimental results at different compositions. We show how the dielectric response can be estimated to a high level of accuracy using three dielectric relaxations: a dominant and slower process at microwave frequencies and two faster processes. A deeper study of the dynamics of the hydrogen bond network formed in these systems reveals how collective processes between the individual species are the origin of the final

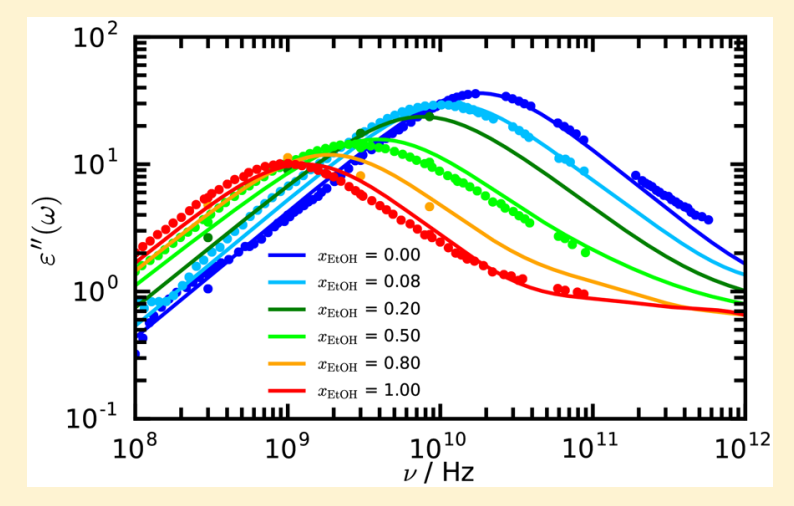
dielectric response. Our results agree with the "wait-and-switch" mechanism, which describes the dynamics of the hydrogen bond network as the combination of two processes: the fast breakage and formation of individual hydrogen bonds and the subsequent reorganization of the entire network once this process becomes energetically favorable. Since the dielectric response is related to dipole reorientations in the system, it is directly linked to these mechanisms.

\section{INTRODUCTION}

The interest in determining dielectric spectra of liquid mixtures resides in studying the dynamics of these systems and identifying possible deviations from the behavior of pure components and their ideal mixtures. The microwave heating efficiency in these types of binary system has been shown to depend significantly on the nonideality of the mixture, where higher heating rates are obtained for compositions at which negative deviations from the ideal solution are observed and intermolecular interactions are weaker. ${ }^{1}$ The amount of experimental work in this area is significant and includes systems such as alcohol/water, ${ }^{2-11}$ glycine/water, ${ }^{12,13} \mathrm{DMSO} /$ alcohol, ${ }^{14}$ and mixtures of acetone, DMSO, and isopropanol. ${ }^{15}$ Other authors have investigated the dielectric response of aqueous solutions of $\alpha$-D-glucose, ${ }^{16,17}$ lysine, ${ }^{18}$ and nucleotides, ${ }^{19}$ mixtures of acrylic esters and alcohols, ${ }^{20}$ ionic liquids in water $^{21}$ and ethanol, ${ }^{22}$ and $p$-fluorophenyl acetonitile/2butylphenol and 2-chlor-6-fluoro-benzaldehyde/o-ethylphenol systems. ${ }^{23}$ Mixtures of polar and nonpolar molecules such as ethanol and benzene have also received some attention in the literature. $^{24-27}$

One of the most studied systems is the ethanol/water mixture. Ethanol molecules are among the smallest molecules to have both hydrophobic and hydrophilic groups. This is why aqueous ethanol solutions are considered as a reference for the study of interactions in biological processes, such as protein folding, ligand binding, or membrane assembly, where hydrophobic hydration has a significant influence. ${ }^{28}$ In addition, ethanol/water mixtures can be found in numerous applications ranging from their use as a solvent, biofuel, or alcoholic beverage. Using microwaves as an alternative heating option for these systems (e.g., for distillation or pervaporation) has also been studied in the past. ${ }^{29-31}$

The concentration and temperature dependence of the dielectric spectrum of this binary system have been analyzed by several authors, ${ }^{2-7,11,32,33}$ including interpretation of the physical origin of the different observed relaxation mechanisms. These mixtures are generally characterized by the presence of two relaxation processes, with a third process appearing at high ethanol concentrations. The first and most intense relaxation has frequently been assigned to the dynamics of the reorganization of the entire hydrogen bond network and is highly dependent on temperature and concentration. Despite the presence of two different components in the mixture, their

Received: December 12, 2017

Revised: January 3, 2018

Published: January 4, 2018 
individual contributions cannot be clearly discerned, which suggests a collective response appearing as a single peak in the spectrum. A second and faster relaxation process would correspond to the breakage and formation of individual hydrogen bonds associated with the reorganization of the entire structure. ${ }^{34}$ Finally, at high ethanol concentrations, an additional intermediate relaxation process has been observed and thought to be related to the chain-like structure of pure ethanol. ${ }^{35,36}$ The presence of water molecules disrupts this chain-like configuration, and this additional relaxation mechanism vanishes as water becomes more predominant in the mixture. In this context, the mechanisms leading to the strengthening or weakening of hydrogen bond networks have been the object of discussion for a long time. ${ }^{37-44} \mathrm{~A}$ consensus seems to have emerged relating to the configuration of the first hydration shell of hydrophobic groups and its influence on the structure of the network. However, the exact mechanism by which this configuration is established is not fully understood. $^{33,45-51}$

Despite the abundant literature based on experimental studies, computational work on the determination of dielectric properties of mixtures is not very common. Nevertheless, some studies have led to the development of both theoretical and empirical mixing rules for the interpolation of dielectric properties of mixtures from their pure constituents. ${ }^{52-56}$ Additionally, Zasetsky et al. determined the dielectric spectrum of methanol/water mixtures in the $\mathrm{GHz} / \mathrm{THz}$ region using molecular dynamics (MD) simulations. ${ }^{57}$ In their study, the authors introduce a method to obtain the distribution of relaxation times from the dipole moment autocorrelation function. Analyzing the individual contribution of the two components of the mixture, they conclude that both exhibit two distinct relaxation modes. Additionally, accurate values of the static dielectric constant of ethanol/water mixtures have also been obtained using molecular dynamics simulations with polarizable force fields. ${ }^{45,46}$ The determination of the dielectric spectrum of ethanol/water mixtures has been mentioned as an example of the potential of molecular dynamics simulations for the estimation of dielectric processes. ${ }^{58}$ However, as far as we are aware, no previous attempt has been carried out to evaluate the accuracy of the prediction of dielectric spectra of ethanol/ water mixtures with respect to the experimental data available in the literature.

In this work, we perform molecular dynamics simulations to study the concentration dependence of the dielectric response of ethanol/water mixtures, and we correlate this response to the dynamics of the hydrogen bond network. The remainder of this paper is organized as follows. In the next section, we provide the details of the molecular dynamics simulations, including a description of the force fields used to represent the intermolecular interactions and details of the analysis of the trajectories to determine properties of the system, such as the dielectric spectra. We then compare the results of the simulations with experimental measurements available from the literature. The effect of concentration on the structure and dynamics of the hydrogen bond network is then studied and correlated with the dielectric response. The results obtained in this work are therefore helpful to reach a better understanding of changes in the dynamics of alcohol/water mixtures with increasing alcohol concentrations and can be useful to model microwave heating profiles of these systems. Finally, we summarize the main conclusions of the work and provide directions for future research.

\section{COMPUTATIONAL DETAILS}

In previous work, ${ }^{59}$ we analyzed the ability of different force fields to predict the dielectric spectra of pure ethanol and water. On the basis of this, we decided to model ethanol by means of the TraPPE-UA force field. ${ }^{60,61}$ For water, we use a modified version of the $\mathrm{Fw}$-SPC model $^{62}$ where bond stretching is suppressed. Flexibility slows a dielectric response, resulting in relaxation times for the $\mathrm{Fw}$-SPC model that are longer than those observed experimentally. By disallowing bond stretching, the dielectric response of the Fw-SPC model is quickened, while preserving its ability to reproduce the static dielectric constant of water to an acceptable level. In addition, compatibility between the modified Fw-SPC model and the TraPPE-UA force field is maintained, in the sense that both are characterized by rigid bonds and flexible angles. The suppression of bond stretching allows the use of longer time steps ( $1 \mathrm{fs}$ ) and reduces the duration of the simulations, while leading to reasonable results, as shown below. Previous studies have used polarizable force fields. ${ }^{45,46}$ However, we consider this level of detail unnecessary for the properties we intend to predict. In fact, by using nonpolarizable force fields, we are able to perform simulations that are 5-10 times longer. This is important for the accurate representation of the dielectric response, since we ensure we are covering enough dipole moment correlation times.

Molecular dynamics (MD) simulations are carried out using GROMACS 4.6.3. ${ }^{63}$ Input files for all simulations performed in this work are openly available from the University of Strathclyde data repository at http://dx.doi.org/10.15129/ 484b40b0-c5d9-42b6-9e64-581bdc710030. We study ethanol/ water mixtures of different concentrations, where all systems consist of simulation boxes containing 1000 molecules (as shown in Table 1) and are simulated for $25 \mathrm{~ns}$. The equations

Table 1. Mole Fractions $\left(x_{\mathrm{EtOH}}\right)$ and Number of Molecules $\left(N_{\mathrm{EtOH}}, N_{\text {water }}\right)$ employed in the Molecular Dynamics Simulations of Ethanol/Water Mixtures

\begin{tabular}{rrr}
$x_{\text {EtOH }}$ & $N_{\text {EtOH }}$ & $N_{\text {water }}$ \\
0.00 & 0 & 1000 \\
0.08 & 80 & 920 \\
0.20 & 200 & 800 \\
0.50 & 500 & 500 \\
0.80 & 800 & 200 \\
1.00 & 1000 & 0 \\
\hline
\end{tabular}

of motion are integrated by means of the leapfrog algorithm ${ }^{64}$ with a time step of 1 fs. The trajectory of the molecules is stored every $100 \mathrm{fs}$, while the energy is recorded every $50 \mathrm{fs}$. The simulations are performed within the NPT ensemble at $298 \mathrm{~K}$ and 1 bar. The Nosé-Hoover thermostat, ${ }^{65,66}$ with a time constant of $0.1 \mathrm{ps}$, is used, while the pressure is controlled by means of a Parrinello-Rahman barostat ${ }^{67,68}$ with a compressibility of $4.5 \times 10^{-5} \mathrm{bar}^{-1}$ and a time constant of 1.0 ps. The LINCS algorithm ${ }^{69}$ is employed to constrain bond lengths. A cutoff radius of $1.4 \mathrm{~nm}$ is used for the short-ranged Lennard-Jones (LJ) interactions. Long-range electrostatics are treated with the particle mesh Ewald (PME) method $^{70}$ with a truncation at the same distance as the LJ cutoff and a spacing for the PME grid of $0.12 \mathrm{~nm}$. Analytical tail corrections in potential energy are used to compensate for the truncation in LJ interactions. Cubic and tinfoil periodic boundary conditions are used in every case, where the system and its periodic images 
are assumed to be immersed in a perfectly conducting medium (infinite dielectric constant). ${ }^{71}$ Starting from independent and equilibrated configurations, four simulations are carried out at every concentration. The results are averaged across those four samples to improve statistics.

Determination of Dielectric Spectrum from MD Simulations. The total dipole moment $\mathbf{M}$ of a polar system can be determined from the sum of the individual dipole moments $\boldsymbol{\mu}_{i}$ of each molecule in the system (i.e., $\mathbf{M}=\sum_{i}^{N} \boldsymbol{\mu}_{i}$ ). The MD simulations are able to generate a time series of this quantity. With this time series, the frequency-dependent dielectric response is directly derived from the fluctuations of the dipole moment: ${ }^{72}$

$$
\frac{\hat{\varepsilon}(\omega)-\varepsilon_{\infty}}{\varepsilon_{0}-\varepsilon_{\infty}}=1+i \omega \hat{\phi}(\omega)
$$

where $\hat{\phi}(\omega)$ corresponds to the Fourier transform of the total dipole moment autocorrelation function $\phi(t)$, which is given by

$$
\phi(t)=\frac{\langle\mathbf{M}(t) \cdot \mathbf{M}(0)\rangle}{\langle\mathbf{M}(0) \cdot \mathbf{M}(0)\rangle}
$$

In eq $1, \varepsilon_{\infty}$ is the infinite frequency relative permittivity, and $\varepsilon_{0}$ is the zero-frequency or static dielectric constant.

In order to determine the frequency-dependent dielectric response from eq 1 , it is necessary to obtain the Fourier transform of the dipole moment autocorrelation function $\phi(t)$. However, this becomes straightforward if we make use of predefined models for dielectric relaxation, such as the Debye (D) and the Havriliak-Negami (HN) models. The combination of $\mathrm{HN}$ and Debye relaxations can be useful in cases in which multiple processes are observed. The expression corresponding to a $1 \mathrm{HN}+1 \mathrm{D}$ model is given by

$$
\frac{\hat{\varepsilon}(\omega)-\varepsilon_{\infty}}{\varepsilon_{0}-\varepsilon_{\infty}}=\frac{A_{1}}{\left[1+\left(-i \omega \tau_{1}\right)^{\alpha}\right]^{\beta}}+\frac{A_{2}}{1-i \omega \tau_{2}}
$$

where $A_{i}$ and $\tau_{i}$ represent the intensity and relaxation time of the different relaxation processes, respectively. $\alpha$ and $\beta$ are parameters related to the broadness and the asymmetry of the $\mathrm{HN}$ signal, respectively.

The real $\hat{\varepsilon}^{\prime}(\omega)$ and imaginary $\hat{\varepsilon}^{\prime \prime}(\omega)$ parts of the dielectric response result in

$$
\begin{aligned}
\hat{\varepsilon}^{\prime}(\omega)= & \varepsilon_{\infty}+\left(\varepsilon_{0}-\varepsilon_{\infty}\right)\left\{A _ { 1 } \left[1-2\left(\omega \tau_{1}\right)^{\alpha} \cos \left(\frac{\pi \alpha}{2}\right)\right.\right. \\
& \left.\left.+\left(\omega \tau_{1}\right)^{2 \alpha}\right]^{-\beta / 2} \cos (-\beta \varphi)+\frac{A_{2}}{1+\omega^{2} \tau_{2}^{2}}\right\} \\
\hat{\varepsilon}^{\prime \prime}(\omega)= & \left(\varepsilon_{0}-\varepsilon_{\infty}\right)\left\{A _ { 1 } \left[1-2\left(\omega \tau_{1}\right)^{\alpha} \cos \left(\frac{\pi \alpha}{2}\right)\right.\right. \\
& \left.\left.+\left(\omega \tau_{1}\right)^{2 \alpha}\right]^{-\beta / 2} \sin (-\beta \varphi)+\frac{A_{2} \omega \tau_{2}}{1+\omega^{2} \tau_{2}^{2}}\right\}
\end{aligned}
$$

with

$$
\varphi=\arctan \left[-\frac{\left(\omega \tau_{1}\right)^{\alpha} \sin (\pi \alpha / 2)}{1-\left(\omega \tau_{1}\right)^{\alpha} \cos (\pi \alpha / 2)}\right]
$$

With the addition of a second Debye relaxation to the model ( 1 $\mathrm{HN}+2 \mathrm{D})$, the frequency-dependent dielectric constant becomes

$$
\frac{\hat{\varepsilon}(\omega)-\varepsilon_{\infty}}{\varepsilon_{0}-\varepsilon_{\infty}}=\frac{A_{1}}{\left[1+\left(-i \omega \tau_{1}\right)^{\alpha}\right]^{\beta}}+\frac{A_{2}}{1-i \omega \tau_{2}}+\frac{A_{3}}{1-i \omega \tau_{3}}
$$

where the real and imaginary parts are given by

$$
\begin{aligned}
\hat{\varepsilon}^{\prime}(\omega)= & \varepsilon_{\infty}+\left(\varepsilon_{0}-\varepsilon_{\infty}\right)\left\{A _ { 1 } \left[1-2\left(\omega \tau_{1}\right)^{\alpha} \cos \left(\frac{\pi \alpha}{2}\right)\right.\right. \\
& \left.\left.+\left(\omega \tau_{1}\right)^{2 \alpha}\right]^{-\beta / 2} \cos (-\beta \varphi)+\frac{A_{2}}{1+\omega^{2} \tau_{2}^{2}}+\frac{A_{3}}{1+\omega^{2} \tau_{3}^{2}}\right\} \\
\hat{\varepsilon}^{\prime \prime}(\omega)= & \left(\varepsilon_{0}-\varepsilon_{\infty}\right)\left\{A_{1}\left[1-2\left(\omega \tau_{1}\right)^{\alpha} \cos \left(\frac{\pi \alpha}{2}\right)+\left(\omega \tau_{1}\right)^{2 \alpha}\right]^{-\beta / 2} \sin (-\beta \varphi)\right. \\
& \left.+\frac{A_{2} \omega \tau_{2}}{1+\omega^{2} \tau_{2}^{2}}+\frac{A_{3} \omega \tau_{3}}{1+\omega^{2} \tau_{3}^{2}}\right\}
\end{aligned}
$$

with

$$
\varphi=\arctan \left[-\frac{\left(\omega \tau_{1}\right)^{\alpha} \sin (\pi \alpha / 2)}{1-\left(\omega \tau_{1}\right)^{\alpha} \cos (\pi \alpha / 2)}\right]
$$

By deriving the time domain representation of these models, we can fit their characteristic parameters to reproduce the dipole moment time autocorrelation functions obtained from MD simulations. Then it is simple to obtain the frequency dependence of the dielectric constant from the frequency domain representation of the models (eqs 4 and 7).

The time representation of the $1 \mathrm{HN}+1 \mathrm{D}$ model results in

$$
\begin{aligned}
\phi(t)= & 1-A_{1} \sum_{k=0}^{\infty}(-1)^{k} \frac{\Gamma(\beta+k)}{\Gamma(1+k)} \frac{\left(t / \tau_{1}\right)^{\alpha(\beta+k)}}{\Gamma(\beta) \Gamma[\alpha(\beta+k)+1]} \\
& -A_{2}\left[1-\exp \left(-\frac{t}{\tau_{2}}\right)\right]
\end{aligned}
$$

while the $1 \mathrm{HN}+2 \mathrm{D}$ model in the time domain is given by

$$
\begin{aligned}
\phi(t)=1 & -A_{1} \sum_{k=0}^{\infty}(-1)^{k} \frac{\Gamma(\beta+k)}{\Gamma(1+k)} \times \frac{\left(t / \tau_{1}\right)^{\alpha(\beta+k)}}{\Gamma(\beta) \Gamma[\alpha(\beta+k)+1]} \\
& -A_{2}\left[1-\exp \left(-\frac{t}{\tau_{2}}\right)\right]-A_{3}\left[1-\exp \left(-\frac{t}{\tau_{3}}\right)\right]
\end{aligned}
$$

where $\Gamma$ is the gamma function: $\Gamma(n)=(n-1)$ !

A more detailed explanation of the procedure used to obtain dielectric spectra from molecular dynamics simulations can be found in a previous publication. ${ }^{59}$

\section{RESULTS AND DISCUSSION}

Thermodynamic Properties. We initially validate our simulations by determining a number of thermodynamic properties such as density, thermal expansion coefficient, isothermal compressibility, and heat capacity of ethanol/water mixtures. In general, the main experimental trends are satisfactorily captured by the force fields employed in this work, as shown in Figure S1 of the Supporting Information.

Dipole Moment Autocorrelation Function in Binary Systems. In previous work, ${ }^{59}$ we limited ourselves to the use of single relaxations in the analysis of the dielectric response of one component systems. However, as mentioned in the introduction, ethanol/water mixtures are generally modeled as the superposition of three relaxation processes. Only in the case of pure water does two relaxation processes suffice to reproduce the response. As an example, in Figure 1, we compare the performance of different models when fitting the dipole 


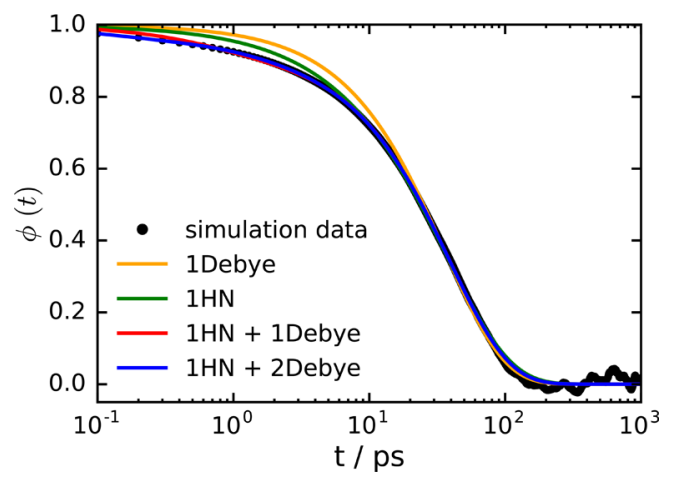

Figure 1. Comparison of the performance of different models used for the fit of dipole moment autocorrelation functions of mixtures. Data in the figure correspond to a simulation performed at $298 \mathrm{~K}$ and 1 bar for an ethanol/water mixture with an ethanol mole fraction of 0.5 . Black circles represent the dipole moment autocorrelation function obtained from simulation. Four models are compared: single Debye (orange), single $\mathrm{HN}$ (green), $1 \mathrm{HN}+1 \mathrm{D}$ (red), and $1 \mathrm{HN}+2 \mathrm{D}$ (blue).

moment autocorrelation function (ACF) of an equimolar ethanol/water mixture at $298 \mathrm{~K}$ and 1 bar. The fitting procedure aims to reduce the sum of squares of the difference between simulation data and model predictions and is performed using the NLopt $\mathrm{C}++$ library with the BOBYQA algorithm. ${ }^{73}$ Only data with a dipole moment ACF greater than 0.1 is considered in the fit to avoid the noisy tails observed below this value.

As expected, the use of a single $\mathrm{HN}$ relaxation provides a better result than a single Debye process. Nevertheless, it is clearly observed how a minimum of two relaxation processes (1 $\mathrm{HN}+1 \mathrm{D}$ model, see eq 9) is required to reproduce accurately the result obtained in our simulations. The additional Debye relaxation is included to take into account fast processes occurring at very short correlation times. Moreover, the introduction of an intermediate Debye relaxation process ( 1 $\mathrm{HN}+2 \mathrm{D}$ model, see eq 10) further improves the accuracy of the fit in that region. This result is consistently observed in the wide range of concentrations we have studied. Therefore, in the following, we use a combination of one Havriliak-Negami and two Debye relaxation processes to fit the dipole moment ACF of ethanol/water mixtures, with the noted exception of pure water, for which adding an intermediate relaxation process does not significantly improve the fit.

Dielectric Response of Ethanol/Water Mixtures from MD Simulations. As discussed in the introduction, the concentration has a significant influence on the dielectric response of binary systems..$^{2-7,11,32,33}$ In Figure 2, we present results for the concentration dependence of the dipole moment ACF of ethanol/water mixtures, which originate from averaging over four independent simulations. The generally low uncertainties indicate a low variability between the different runs for this particular system. As mentioned above, a $1 \mathrm{HN}+1$ $\mathrm{D}$ model is used for pure water, while a $1 \mathrm{HN}+2 \mathrm{D}$ fit is used when ethanol is present in the mixture. On the basis of Figure 2 , we realize that the initial relaxation at short times is practically independent of composition. However, the influence of concentration becomes clear when we focus on the main relaxation process. Although it is not easily appreciated, an intermediate and less intense process is also captured by the model. The influence of concentration on the different relaxation parameters given by these models is summarized in Table 2 and Figures 3, 4, and 5.

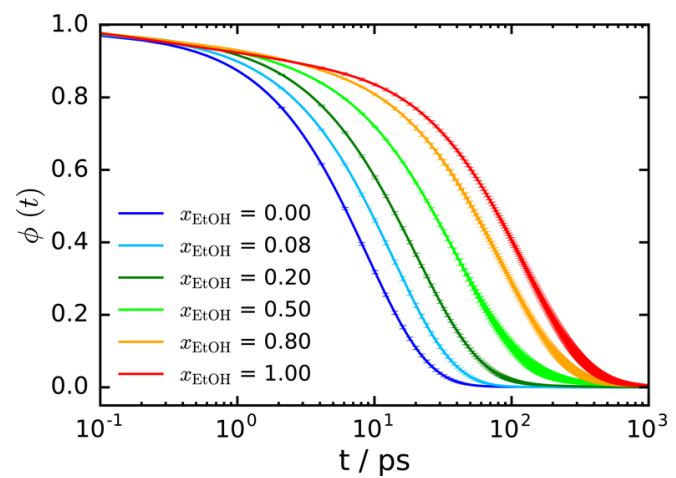

Figure 2. Evolution of the dipole moment autocorrelation function with composition in ethanol/water mixtures at $298 \mathrm{~K}$ and 1 bar.

The first aspect to note in Figure 3 is the good agreement between the main relaxation time $\tau_{1}$ obtained from our simulations and the experimental data. A slight underprediction is observed, particularly at intermediate ethanol compositions, but the trend in the data is equivalent. Small differences can be attributed to the fact that most experimental studies have not used a $1 \mathrm{HN}+2 \mathrm{D}$ model. Some model the main relaxation by means of the Debye model or use a lower number of relaxations. In any case, this represents a significant achievement, particularly in terms of being able to predict microwave heating rates, since this process is dominated by this relaxation. According to the literature, ${ }^{3-7,11,32,33}$ this first and most intense relaxation $\left(\tau_{1} \approx 8-200 \mathrm{ps}\right)$ corresponds to reorganization of the hydrogen bond network and is generally modeled as a Cole-Cole or Cole-Davidson relaxation. A fact that may appear counterintuitive is the increase of relaxation time with a growing ethanol concentration. Water has a stronger hydrogen bonding structure than ethanol, and one could think that dipole reorientation in highly aqueous systems might be more constrained. ${ }^{11}$ In this context, a "wait-and-switch" model ${ }^{34}$ has been suggested to describe the dynamics of the liquid phase. According to this mechanism, a "waiting" time $\left(\tau_{1}\right)$ is required for the hydrogen bond network to reorganize and find the next available site to "switch" a hydrogen bond $\left(\tau_{2}\right)$. It is known that oscillations of free $\mathrm{OH}$ groups, related to the formation and breakage of hydrogen bonds, appear as fast relaxation processes in the spectrum $\left(\tau_{2} \leq 2 \mathrm{ps}\right)$. However, the availability of sites to form new hydrogen bonds is limited. The increase of the water concentration in the mixture results in an increasing probability of finding hydrogen bonding sites, which in turn decreases the potential energy barrier between two dipolar orientations. Therefore, the activation energy required to perform a molecular rotation can be more easily overcome in mixtures with a higher water content, resulting in shorter "waiting" times, $\tau_{1} \cdot{ }^{11}$ As a result, more frequent modifications of the hydrogen bond structure will occur, leading to the faster decays and shorter dielectric relaxation times as the mixture approaches pure water. Additionally, according to the experimental results, two distinct, nearly linear regions (in a logarithmic scale) can be observed and intersect at a mole fraction of approximately 0.2 , as previously noted by Bao et al. ${ }^{3}$ Despite the reduced number of simulation results, this feature seems to be captured by the force fields used in this work. This mole fraction has previously been reported as a turning point for several processes in ethanol/water mixtures. Below this concentration, ethanol molecules are believed to disrupt circular clusters commonly observed in pure water, at the 
Table 2. Dielectric Relaxation Parameters for Ethanol/Water Mixtures ${ }^{a}$

\begin{tabular}{|c|c|c|c|c|c|c|}
\hline$x_{\mathrm{EtOH}}$ & 0.00 & 0.08 & 0.20 & 0.50 & 0.80 & 1.00 \\
\hline$\tau_{1} / \mathrm{ps}$ & $8.93 \pm 0.10$ & $13.8 \pm 0.3$ & $21.3 \pm 0.6$ & $41.3 \pm 0.7$ & $85 \pm 3$ & $125 \pm 3$ \\
\hline$A_{1}$ & $0.981 \pm 0.004$ & $0.959 \pm 0.005$ & $0.95 \pm 0.02$ & $0.943 \pm 0.008$ & $0.920 \pm 0.006$ & $0.910 \pm 0.005$ \\
\hline$\alpha$ & $0.990 \pm 0.004$ & $0.992 \pm 0.003$ & $0.97 \pm 0.02$ & $0.94 \pm 0.03$ & $0.97 \pm 0.02$ & $0.98 \pm 0.02$ \\
\hline$\beta$ & 1.00 & 1.00 & $0.99 \pm 0.02$ & 1.00 & 1.00 & 1.00 \\
\hline$\tau_{2} / \mathrm{ps}$ & & $1.3 \pm 0.4$ & $1.3 \pm 1.0$ & $1.0 \pm 0.4$ & $1.5 \pm 0.4$ & $1.4 \pm 0.4$ \\
\hline $\mathrm{A}_{2}$ & & $0.018 \pm 0.004$ & $0.03 \pm 0.02$ & $0.032 \pm 0.004$ & $0.046 \pm 0.004$ & $0.045 \pm 0.001$ \\
\hline $\mathrm{A}_{3}$ & $0.019 \pm 0.001$ & $0.023 \pm 0.001$ & $0.016 \pm 0.004$ & $0.025 \pm 0.004$ & $0.034 \pm 0.002$ & $0.045 \pm 0.004$ \\
\hline$\varepsilon_{0}$ & 78.4 & 65.6 & 55.1 & 39.2 & 29.4 & 24.7 \\
\hline$\varepsilon_{0 \operatorname{sim}}$ & $76.0 \pm 0.7$ & $62.8 \pm 0.2$ & $49.9 \pm 0.4$ & $29.2 \pm 0.3$ & $21.6 \pm 0.5$ & $17.7 \pm 0.4$ \\
\hline$\varepsilon_{\infty}$ & 3.35 & 3.24 & 3.08 & 2.68 & 2.27 & 2.00 \\
\hline
\end{tabular}

${ }^{a}$ At $298 \mathrm{~K}$ and 1 bar. The experimental values of the static dielectric constant $\left(\varepsilon_{0}\right)$ have been obtained from different sources. ${ }^{2,4,5,7}$

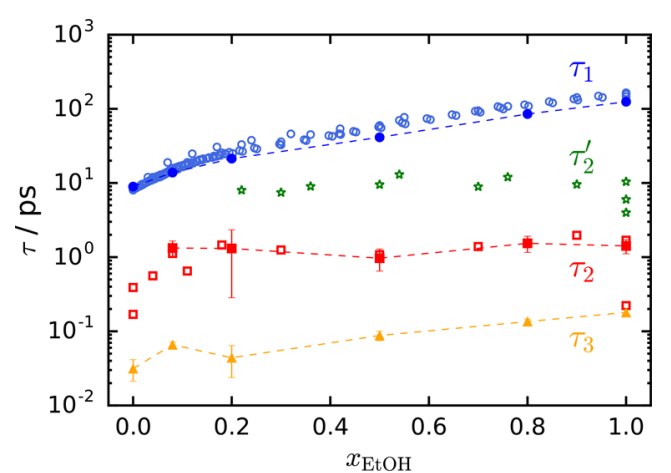

Figure 3. Variation of relaxation times with concentration in ethanol/ water mixtures at $298 \mathrm{~K}$ and 1 bar. Circles represent the relaxation times of the dominant HN relaxation (1), while stars, squares, and triangles correspond to the intermediate $\left(2^{\prime}\right)$, fast $(2)$, and very fast (3) processes, respectively. Filled symbols correspond to the fit of the $1 \mathrm{HN}+2 \mathrm{D}$ model to simulation data. In the case of pure water $\left(x_{\mathrm{EtOH}}\right.$ $=0)$, a $1 \mathrm{HN}+1 \mathrm{D}$ model is used. Open symbols correspond to experimental data obtained from different sources: $\tau_{1}{ }^{3-6,11,33} \tau_{2}^{\prime},{ }^{4,5,33}$ and $\tau_{2}{ }^{5,33}$ The dashed lines are a guide to the eye.

price of slowing down the dielectric response. ${ }^{3}$ For higher concentrations, self-interactions between ethanol molecules lead to a different organization of the hydrogen bond network. In addition, around this concentration $\left(x_{\mathrm{EtOH}}=0.2\right)$ both activation enthalpy and activation entropy of the dominant relaxation process reach their maximum value, and the shear viscosity exhibits a relative minimum. ${ }^{4,6}$

Our simulations fully capture the fast relaxations associated with the formation and breakage of hydrogen bonds, with relaxation times $\tau_{2} \approx 1-2$ ps. However, it was not possible to discern an additional intermediate process $\left(\tau_{2}^{\prime} \approx 7-12 \mathrm{ps}\right)$, as seen in experimental studies and related to the formation of ethanol chains at higher ethanol concentrations. ${ }^{4,5,33,35,36}$ It is possible that the force fields employed in this work are not able to reproduce this phenomenon. This additional intermediate relaxation process is thought to arise from the fluctuations of terminal single hydrogen-bonded ethanol monomers of the chain structure $\left(\tau_{2}^{\prime} \approx 7-12 \mathrm{ps}\right)$. As water molecules are introduced in the mixture, they tend to infiltrate the ethanol chain structure, giving rise to shorter chains and, therefore, increasing the number of terminal ethanol molecules. This results in a more intense intermediate relaxation process with a growing fraction of water in the mixture. However, when an approximately equimolar mixture is reached, the speed-up of the main relaxation process $\left(\tau_{1}\right)$ results in an overlap with this

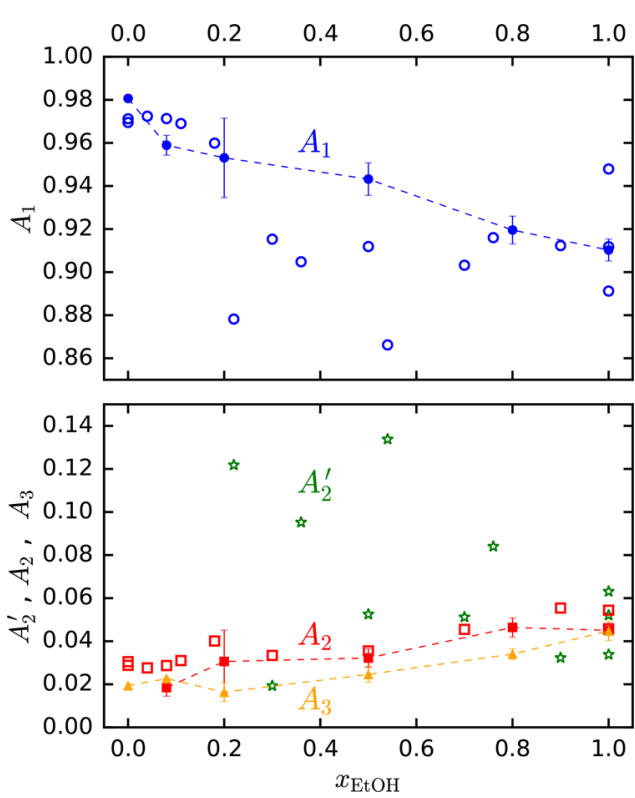

Figure 4. Variation of relaxation intensities with concentration in ethanol/water mixtures at $298 \mathrm{~K}$ and 1 bar. Circles represent the relaxation intensities of the dominant $\mathrm{HN}$ relaxation (1), while stars, squares, and triangles correspond to the intermediate $\left(2^{\prime}\right)$, fast (2), and very fast (3) processes, respectively. Filled symbols correspond to the fit of the $1 \mathrm{HN}+2 \mathrm{D}$ model to simulation data. In the case of pure water $\left(x_{\mathrm{EtOH}}=0\right)$, a $1 \mathrm{HN}+1 \mathrm{D}$ model is used. Open symbols correspond to experimental data obtained from different sources: $A_{1}{ }^{4,5,33} A_{2}^{\prime},{ }^{4,5,33}$ and $A_{2}{ }^{5,33}$ The dashed lines are a guide to the eye.

intermediate mode $\left(\tau_{2}^{\prime}\right)$, and its contribution is difficult to observe experimentally. Both the intermediate $\left(\tau_{2}^{\prime}\right)$ and the fast $\left(\tau_{2}\right)$ processes can generally be modeled by means of simple Debye relaxations.

Finally, the accurate fit of the dipole moment ACFs generated through simulations requires an even faster process $\left(\tau_{3} \approx 0.02-0.2 \mathrm{ps}\right)$. Since these values are greater than the time step used in our simulations ( $1 \mathrm{fs}$ ), but less than the trajectory sampling times $(0.1 \mathrm{ps})$, this process cannot be attributed to any physical mechanism captured by the simulations and could be the result of the contribution of several high frequency phenomena. Nevertheless, Li et al. ${ }^{33}$ observed relaxation times, $\tau_{4}$, in this same order of magnitude for pure ethanol.

With the analyisis of the intensity of the different relaxation processes, the relative contribution of the main process $\left(A_{1}\right)$ is significantly larger than that of faster processes. The larger magnitude of this relaxation process, which corresponds to the 


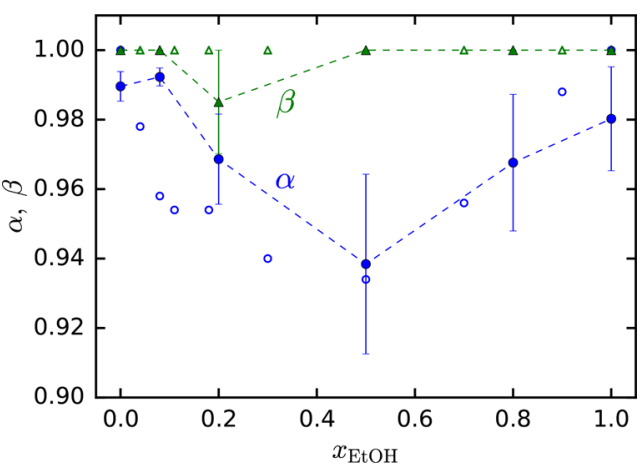

Figure 5. Variation of the Havriliak-Negami parameters $\alpha$ and $\beta$ with concentration in ethanol/water mixtures at $298 \mathrm{~K}$ and 1 bar. Circles represent values of parameter $\alpha$, while triangles correspond to parameter $\beta$. Filled symbols correspond to the fit of the $1 \mathrm{HN}+2$ $\mathrm{D}$ model to simulation data. In the case of pure water $\left(x_{\mathrm{EtOH}}=0\right)$, a 1 $\mathrm{HN}+1 \mathrm{D}$ model is used. Open symbols correspond to experimental data. ${ }^{5}$ The dashed lines are a guide to the eye.

reorganization of the entire hydrogen bond network, ${ }^{4}$ is explained by the fact that the majority of the molecules in the mixture form part of an extensive network, through multiple hydrogen bond interactions. ${ }^{35}$ Although less intense, faster processes seem to gain more importance as the concentration of ethanol in the mixture increases (see Figure 4). In spite of the fact that experimental data are somewhat scattered, the same trend appears for the relaxation processes we are able to reproduce $\left(A_{1}\right.$ and $\left.A_{2}\right)$.

The symmetric and asymmetric deviations from the Debye model, given by the Havriliak-Negami parameters $\alpha$ and $\beta$, are both relatively weak. In the case of $\alpha$, a minimum is observed for concentrations close to the equimolar mixture, in correspondence with experimental results. ${ }^{5}$ This could have implications on the ideality of the mixture at those concentrations. Another important feature to consider is the nearly Debye behavior of the main relaxation of pure ethanol ( $\alpha=0.98$ and $\beta=1.00$, see Table 2$)$. This differs significantly from results obtained previously, ${ }^{59}$ where a single $\mathrm{HN}$ relaxation was used to fit the simulation data $(\alpha=1.00$ and $\beta$ $=0.77$ ). This suggests that the asymmetric broadening in the spectra observed for a single relaxation fit was in fact due to the presence of additional relaxation processes at higher frequencies.
In summary, we conclude that, although our results require the same number of relaxation processes as the most elaborate experimental fits, ${ }^{4,5,33}$ the intermediate relaxation $\left(\tau_{2}^{\prime}\right)$ is not captured by our models, and a very fast process $\left(\tau_{3}\right)$ appears, with an uncertain physical mechanism. Even so, the excellent results obtained for the main relaxation process $\left(\tau_{1}\right)$ and the fast process $\left(\tau_{2}\right)$ allow for further interpretation of the results in terms of the dynamics of the hydrogen bond network.

In order to obtain an accurate representation of the dielectric spectra of ethanol/water mixtures from our simulations, we need a good estimate of the static dielectric constant and the infinite frequency permittivity. Previous work has shown that the static dielectric constant $\left(\varepsilon_{0}\right)$ is difficult to determine accurately through MD simulations. ${ }^{59,74-76}$ In Figure 6a, we compare our results with available experimental data. ${ }^{2,4,5,7}$ Even though the simulations provide a relatively good prediction, we decided to use the experimental static dielectric constant instead, due to the deviations observed toward higher ethanol concentrations. However, the value of this magnitude for some of the concentrations we have considered in our simulations is unknown. Therefore, we decided to perform a stretched exponential fit through the available data to obtain the missing values:

$$
\varepsilon_{0}=A \exp \left[-\left(\frac{x_{E t O H}}{\tau_{\varepsilon_{0}}}\right)^{\gamma}\right]
$$

with $A=78.4, \tau_{\varepsilon_{0}}=0.821$, and $\gamma=0.740$. The fit was constrained to pass through the static dielectric constant of pure components, which are known with a high level of certainty. In the case of the infinite frequency permittivity $\left(\varepsilon_{\infty}\right)$, the available data are very scattered, as shown in Figure $6 \mathrm{~b}$. Most values provided in the literature originate from fitting experimentally determined dielectric spectra to the Debye and the $\mathrm{HN}$ models. In this work, we select the results given by $\mathrm{Li}$ et al. ${ }^{33}$ for pure ethanol $\left(\varepsilon_{\infty}=2.00\right)$ and pure water $\left(\varepsilon_{\infty}=3.35\right)$ and use a linear interpolation for intermediate concentrations. These authors use the 3 and $2 \mathrm{D}$ relaxation models for ethanol and water, respectively, which is a similar strategy to the $1 \mathrm{HN}$ $+2 \mathrm{D}$ and $1 \mathrm{HN}+1 \mathrm{D}$ models we employ.

The values of $\varepsilon_{0}$ and $\varepsilon_{\infty}$ obtained according to this procedure, for the concentrations used in this work, are shown in Table 2. With this in mind, and using the relaxation
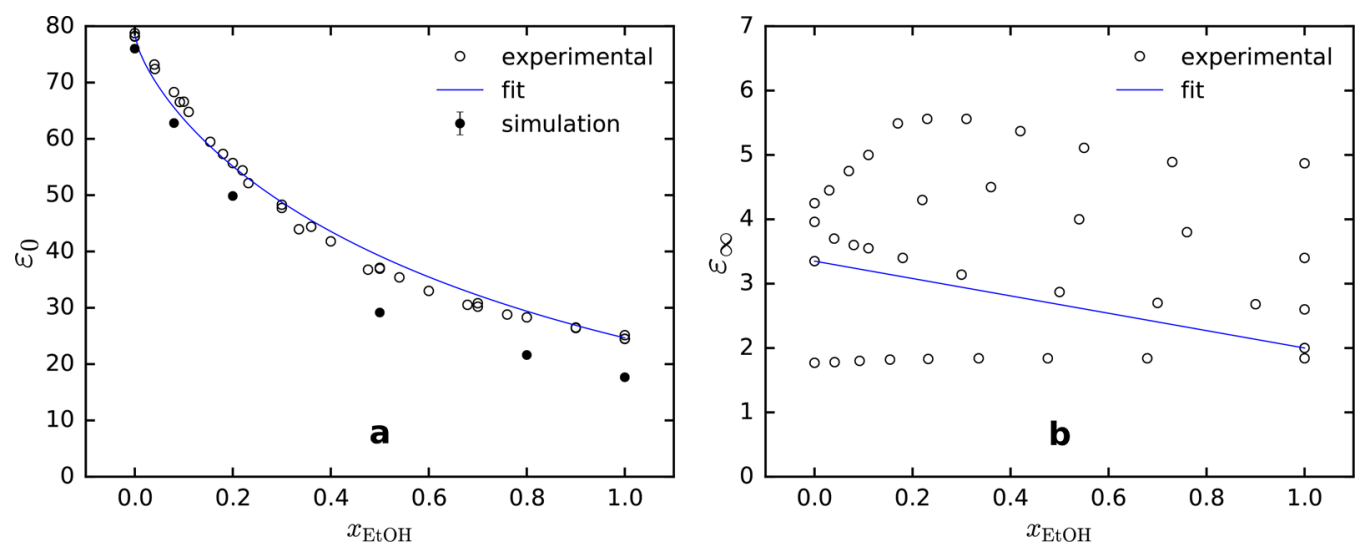

Figure 6. Influence of concentration on (a) the static dielectric constant $\left(\varepsilon_{0}\right)$ and (b) the infinite frequency permittivity $\left(\varepsilon_{\infty}\right)$ of ethanol/water mixtures, at $298 \mathrm{~K}$ and 1 bar. Filled circles represent results obtained in our simulations. Open symbols correspond to experimental data for the static dielectric constant $t^{2,4,5,7}$ and infinite frequency permittivity. ${ }^{3-5,7,33}$ Solid lines show fits to experimental data. 
parameters summarized in Table 2, we proceed to calculate the dielectric spectra of ethanol/water mixtures. As previously stated, a $1 \mathrm{HN}+1 \mathrm{D}$ model (eq 4) was used for pure water, while a $1 \mathrm{HN}+2 \mathrm{D}$ model (eq 7) was employed for the remaining systems. The resulting real and imaginary parts of the spectra are shown in Figure $7 \mathrm{a}, \mathrm{b}$, respectively, and compared with experimental data.
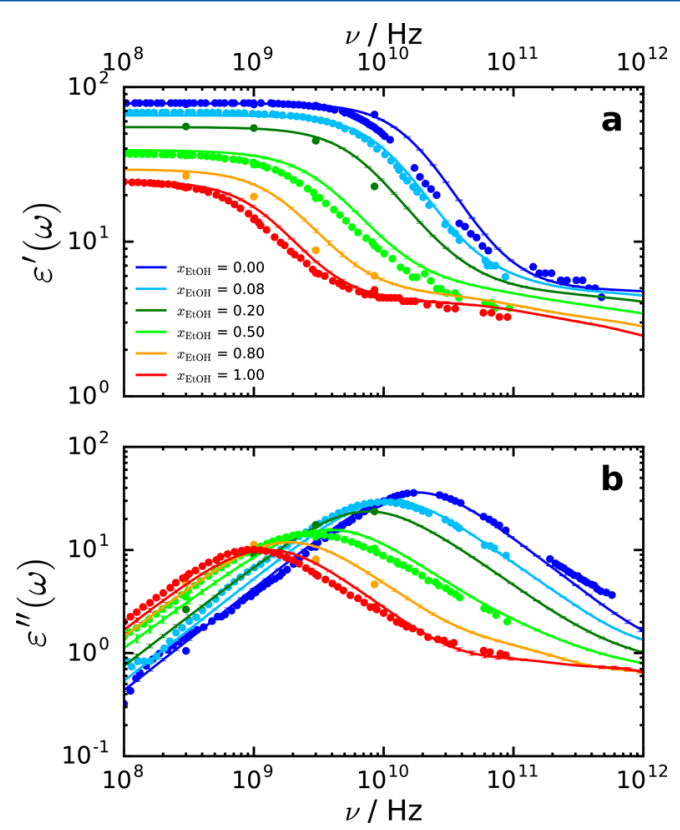

Figure 7. Evolution of the (a) real part and (b) imaginary part of the dielectric spectra of ethanol/water mixtures with composition at $298 \mathrm{~K}$ and 1 bar. Solid lines represent results obtained in our simulations, while circles represent experimental values obtained from different sources. $^{2,5}$

We can observe how the prediction of the dielectric spectra of ethanol/water mixtures is outstanding, especially at low ethanol concentrations. In particular, the dielectric loss of pure water is extremely well-described by the semiflexible Fw-SPC model used in this work (see Figure $7 \mathrm{~b}$ ), outperforming the original Fw-SPC model, which was shown in previous work. ${ }^{59}$ Small deviations appear as the concentration of ethanol in the mixture increases. However, the results are still acceptable in those cases. As explained previously, the global dielectric response of the mixture slows down and becomes less intense as ethanol becomes the dominant component.

Contribution of the Different Relaxation Processes. As a first illustration of the ability of our approach to improve the physical understanding of dielectric responses, we study the decomposition of the dielectric spectra into different relaxation processes. Figure 8 shows the contribution of these processes to the global response for all the concentrations considered in this work. The parameters defining these processes, and their variation with mole fraction, have been reported previously in Table 2 and Figures 3, 4, and 5. In this representation, it becomes clear how the different relaxation mechanisms cannot be individually assigned to specific components of the mixture. If we focus on the main relaxation $\left(\tau_{1}\right)$, only one dominant peak is observed in the spectra, and it is not formed by separate contributions of processes with the frequencies and amplitudes that correspond to the pure components. This reinforces the hypothesis that the mechanism behind the dominant relaxation originates from the global reorganization of the entire structure of the fluid. The intensity of this process is significantly larger than that of the remaining faster processes because more molecules are involved in the response. ${ }^{35}$ The relaxation mechanism shifts as a whole toward lower frequencies as the concentration of ethanol in the mixture increases (from Figure 8 a to Figure $8 \mathrm{f}$ ), indicating that, as expected, the composition of the mixture is an important factor in the dynamics of the hydrogen bond network. As mentioned before, we do not observe an intermediate relaxation process $\left(\tau_{2}^{\prime}\right)$, which had been associated with the presence of chain-like structures formed by ethanol molecules. ${ }^{35,36}$ However, we are able to detect two fast processes $\left(\tau_{2}\right.$ and $\left.\tau_{3}\right)$ that have previously been identified in the literature as the breakage and formation of individual hydrogen bonds. ${ }^{11,34}$

Hydrogen Bond Network. The "wait-and-switch" mechanism $^{34}$ mentioned previously can be used to link the observations on the dynamics of the hydrogen bond network with the dielectric response of ethanol/water mixtures. We have analyzed the hydrogen bond networks in our simulations. Details are provided in the Supporting Information, along with a discussion of their structure. In particular, we find that the hydrogen bond network is more extensive in water than in ethanol. For pure water, there is a hydrogen bond network that spans the system. Significant numbers of water molecules, however, are not bonded to this main network and form monomers, dimers, and a series of smaller networks. As ethanol is added to the mixture, the spanning network seems to gradually vanish. Initially, individual ethanol molecules tend to form hydrogen bonds with water molecules, and the weakening of the hydrogen bond network is not too significant. However, when the mole fraction of ethanol reaches values between 0.08 and 0.20 , the ethanol molecules have more chances to cluster with each other. When this occurs, the size of the network decreases significantly faster due to the hydrophobic nature of those clusters, in agreement with the conclusions reached by $\mathrm{Li}$ et $\mathrm{al}^{33}$ The extended network finally disappears at an ethanol mole fraction between 0.50 and 0.80 . At this point, monomers, dimers, and smaller clusters are more likely to occur. The fraction of both ethanol and water molecules belonging to extended networks increases as the network grows larger, confirming the hypothesis that the dynamics of the hydrogen bond network is the result of the combined action of both species of the mixture, which in turn has implications on the characteristics of the dielectric response of the system.

The lower availability of hydrogen bonding sites at high ethanol concentrations decreases the probability of dipole reorientation in the system. Hence, the dielectric response will be slower. As the mole fraction of water increases, more hydrogen bonding sites become available, reducing the potential energy barrier for dipole reorientation. This generates a more frequent "switch" of the fluid structure and, therefore, a faster dielectric response at higher water concentrations.

Figure 9 shows the effect of ethanol concentration on the distribution of hydrogen bond lifetimes, plotted as a probability density function. As expected from previous studies, $^{4-6,11,33,77-81}$ most hydrogen bonds have a life expectancy shorter than 2 ps. However, increasing ethanol concentrations result in wider distributions extending to longer hydrogen bond lifetimes. This confirms the previous hypothesis since it means that fast hydrogen bond "switches" are less frequent and that the "waiting" time for the reorganization of the hydrogen bond network becomes longer. This is related to the slow down of 

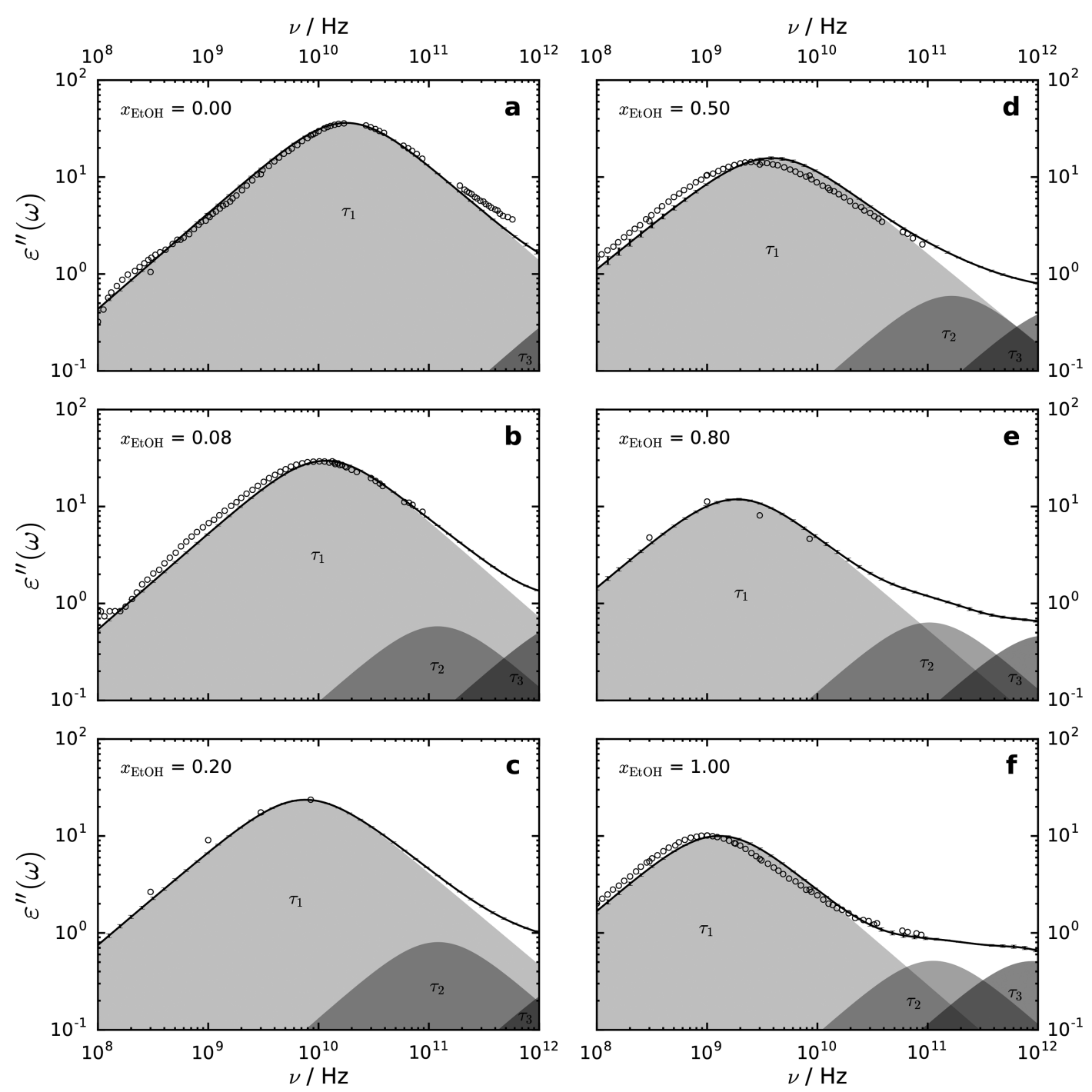

Figure 8. Dielectric spectra of ethanol/water mixtures at $298 \mathrm{~K}$ and 1 bar at mole fractions of ethanol of (a) 0.00, (b) 0.08, (c) 0.20, (d) 0.50, (e) 0.80 , and (f) 1.00. The global response obtained through MD simulations is shown as solid lines. The contribution of different relaxation processes to the dielectric spectra is shown as shaded regions in the figure. Open circles represent experimental values obtained from different sources. ${ }^{2,5}$

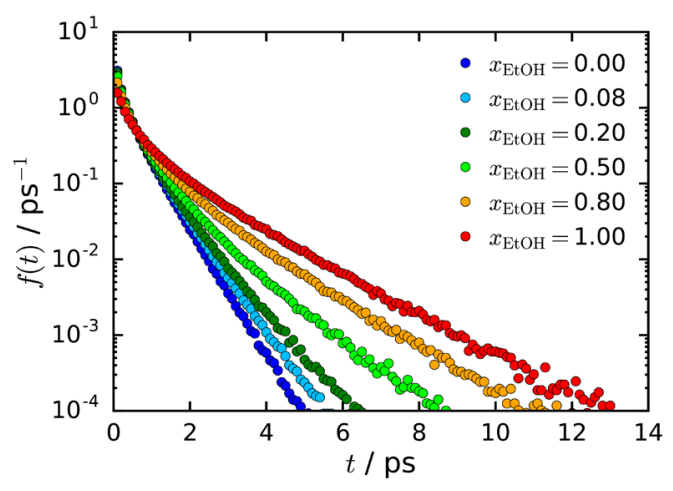

Figure 9. Effect of concentration on the distribution of hydrogen bond lifetimes in ethanol/water mixtures at $298 \mathrm{~K}$ and $1 \mathrm{bar}$.

the global dielectric response at higher ethanol concentrations $\left(\tau_{1}\right.$ in Figure 3$)$.

\section{CONCLUSIONS}

In this work, we use MD simulations to predict the dielectric spectra of ethanol/water mixtures, one of the most studied systems due to their simplicity and wide range of applications. MD simulations are performed using the TraPPE-UA force field for ethanol and a modified version of the Fw-SPC water model. Following the methodology previously applied to the study of single component systems, we are able to predict the dielectric spectra of ethanol/water mixtures of different concentrations to a high level of accuracy. This is an important result, since it allows estimation of the dielectric response, with a good level of confidence, at additional intermediate concentrations that have not been determined experimentally. In addition, it opens the possibility to study the influence on the dielectric response of additional variables, such as temperature. We show how, when ethanol is present in the mixture, at least three relaxation processes are required to model the dielectric response: a dominant process at microwave frequencies and two additional faster processes. Only in the case of water, two relaxation processes suffice. Although we implemented a Havriliak-Negami model to reproduce the dominant process, the deviations from the Debye model are not very substantial, and a rather ideal response is observed. 
Previous studies have suggested that the dielectric response of ethanol/water mixtures originates from collective processes of its extended hydrogen bond network, rather than from individual contributions of the constituents of the mixture. A "wait-and-switch" mechanism ${ }^{34}$ has been proposed, where the dominant and slower relaxation process results from the reorganization of the entire hydrogen bond network which, in turn, requires the breakage and formation of individual hydrogen bonds at a faster rate. The results obtained in this work confirm the predictions of the "wait-and-switch" hypothesis for the ethanol/water mixture. We show how the "switching" time of individual hydrogen bonds increases as the fraction of ethanol in the mixture becomes predominant. A percolating network seems to exist at high water concentrations where the large availability of hydrogen bonds makes the "switches" more frequent and lowers the energy barrier for the reorganization of the entire network. As ethanol becomes predominant in the mixture, the hydrogen bond network tends to vanish. This results in a slower reorganization of the system and, therefore, in a slower global dielectric response at higher ethanol concentrations.

The results presented in this work can easily be extended to other mixtures and offer guidance for systems with limited availability of experimental data, provided accurate and transferable force fields exist for those systems. In addition, the use of MD simulations can provide an even deeper level of understanding of the mechanisms governing fluid mixtures by studying the dynamics of the individual species of the mixture and the correlations between them. The outcomes obtained from MD simulations, that might not be accessible through current experimental methods, can have significant applications in fields such as microwave heating, fluid mixing, separation technologies, and reaction processes in the liquid phase. Additionally, a range of monitoring techniques could potentially be developed based on the conclusions of this and similar studies.

\section{ASSOCIATED CONTENT}

\section{S Supporting Information}

The Supporting Information is available free of charge on the ACS Publications website at DOI: 10.1021/acs.jpcb.7b12220.

Effect of concentration on the thermodynamic properties, the cluster size distribution, the fraction of molecules belonging to extended networks, the distribution of the number of hydrogen bonds formed by each molecule, and the average number of hydrogen bonds formed by each molecule in ethanol/water mixtures at $298 \mathrm{~K}$ and 1 bar (PDF)

\section{AUTHOR INFORMATION}

\section{Corresponding Author}

*E-mail: j.cardona-amengual@strath.ac.uk. Phone: +44 (0)141 4447321.

\section{ORCID}

Javier Cardona: 0000-0002-9284-1899

\section{Notes}

The authors declare no competing financial interest.

\section{ACKNOWLEDGMENTS}

The authors gratefully acknowledge the support of the Department of Chemical and Process Engineering at the
University of Strathclyde and the School of Engineering at the University of Edinburgh, as well as the Engineering and Physical Sciences Research Council (EPSRC). Results were obtained using the EPSRC funded ARCHIE-WeSt High Performance Computer (www.archie-west.ac.uk EPSRC grant no. EP/K000586/1).

\section{REFERENCES}

(1) Sumi, T.; Dillert, R.; Horikoshi, S. Novel Microwave Thermodynamic Model for Alcohol with Clustering Structure in Nonpolar Solution. J. Phys. Chem. B 2015, 119, 14479-14485.

(2) Buck, D. E. The Dielectric Spectra of Ethanol-Water Mixtures in the Microwave Region. Ph.D. Thesis, Massachusetts Institute of Technology, Dept. of Chemistry, 1965.

(3) Bao, J.-Z.; Swicord, M. L.; Davis, C. C. Microwave Dielectric Characterization of Binary Mixtures of Water, Methanol, and Ethanol. J. Chem. Phys. 1996, 104, 4441-4450.

(4) Petong, P.; Pottel, R; Kaatze, U. Water-Ethanol Mixtures at Different Compositions and Temperatures. A Dieletric Relaxation Study. J. Phys. Chem. A 2000, 104, 7420-7428.

(5) Sato, T.; Buchner, R. Dielectric Relaxation Processes in Ethanol/ Water Mixtures. J. Phys. Chem. A 2004, 108, 5007-5015.

(6) Sato, T.; Buchner, R. Cooperative and Molecular Dynamics of Alcohol/Water Mixtures: The View of Dielectric Spectroscopy. J. Mol. Liq. 2005, 117, 23-31.

(7) Sengwa, R.; Madhvi; Sankhla, S.; Sharma, S. Characterization of Heterogeneous Interaction Behavior in Ternary Mixtures by a Dielectric Analysis: Equi-Molar H-bonded Binary Polar Mixtures in Aqueous Solutions. J. Solution Chem. 2006, 35, 1037-1055.

(8) Megriche, A.; Belhadj, A.; Mgaidi, A. Microwave Dielectric Properties of Binary Solvent Water-Alcohol, Alcohol-Alcohol Mixtures at Temperatures between $-35^{\circ} \mathrm{C}$ and $+35^{\circ} \mathrm{C}$ and Dielectric Relaxation Studies. Mediterr. J. Chem. 2011, 1, 200-209.

(9) Singh, A.; Nair, G. R.; Liplap, P.; Gariepy, Y.; Orsat, V.; Raghavan, V. Effect of Dielectric Properties of a Solvent-Water Mixture Used in Microwave-Assisted Extraction of Antioxidants from Potato Peels. Antioxidants 2014, 3, 99-113.

(10) Wei-Na, L.; Jun-Hong, W. Effective Permittivity of Alcohol + Water Mixtures as Influenced by Concentration. J. Chem. Pharm. Res. 2014, 6, 1432-1434.

(11) Dzida, M.; Kaatze, U. Compressibility and Dielectric Relaxation of Mixtures of Water with Monohydroxy Alcohols. J. Phys. Chem. B 2015, 119, 12480-12489.

(12) Sato, T.; Buchner, R.; Fernandez, S.; Chiba, A.; Kunz, W. Dielectric Relaxation Spectroscopy of Aqueous Amino acid Solutions: Dynamics and Interactions in Aqueous Glycine. J. Mol. Liq. 2005, 117, 93-98.

(13) Talware, R.; Hudge, P.; Joshi, Y.; Kumbharkhane, A. Dielectric Relaxation Study of Glycine-Water Mixtures using Time Domain Reflectometry Technique. Phys. Chem. Liq. 2012, 50, 102-112.

(14) Guo-Zhu, J.; Jie, Q. Dielectric Constant of Dimethyl Sulfoxide Monohydric Alcohol Mixture Solution at the Microwave Frequency. Fluid Phase Equilib. 2014, 365, 5-10.

(15) Kaatze, U.; Lönnecke-Gabel, V. Dielectric Spectroscopy on Binary Mixtures of Organic Solvents. J. Mol. Liq. 1991, 48, 45-60.

(16) Liao, X.; Raghavan, G.; Yaylayan, V. Dielectric Properties of Aqueous Solutions of $\alpha$-D-glucose at $915 \mathrm{MHz}$. J. Mol. Liq. 2002, 100, 199-205.

(17) Liao, X.; Raghavan, G.; Dai, J.; Yaylayan, V. Dielectric Properties of $\alpha$-D-glucose Aqueous Solutions at $2450 \mathrm{MHz}$. Food Res. Int. 2003, $36,485-490$.

(18) Liao, X.; Raghavan, G.; Wu, G.; Yaylayan, V. Dielectric Properties of Lysine Aqueous Solutions at $2450 \mathrm{MHz}$. J. Mol. Liq. 2003, 107, 15-19.

(19) Glancy, P.; Beyermann, W. P. Dielectric Properties of Fully Hydrated Nucleotides in the Terahertz Frequency Range. J. Chem. Phys. 2010, 132, 245102. 
(20) Dharmalingam, K.; Ramachandran, K.; Sivagurunathan, P.; Prabhakar Undre, B.; Khirade, P. W.; Mehrotra, S. C. Dielectric Relaxation of Binary Mixtures of Alcohols with Acrylic Esters. J. Appl. Polym. Sci. 2008, 107, 2312-2316.

(21) Koeberg, M.; Wu, C.-C.; Kim, D.; Bonn, M. THz Dielectric Relaxation of Ionic Liquid:Water Mixtures. Chem. Phys. Lett. 2007, 439, 60-64.

(22) Ma, L.; Zhao, K. Dielectric Relaxation Spectroscopy for the Binary System of 1-butyl-3-methylimidazolium Hexafluorophosphate and Ethanol: Interactions and Micro Phase Behavior. RSC Adv. 2012, 2, 10007-10014.

(23) Vasan, S.; Sannaninganavar, F.; Ayachit, N.; Deshpande, D. Dielectric Relaxation in Relation to Viscosity of some Organic Molecules and some of their Binary Mixtures. J. Mol. Liq. 2007, 135, $115-119$.

(24) King, J. F.; Patrick, W. A. The Measurement of Dielectric Constants. J. Am. Chem. Soc. 1921, 43, 1835-1843.

(25) Goss, F. R. 173. The Magnitude of the Solvent Effect in DipoleMoment Measurements. Part III. Polarisation and Association of Alcohols in the Liquid Phase. J. Chem. Soc. 1940, 0, 888-894.

(26) Rätzsch, M. T.; Wohlfarth, C.; Claudius, M. Kirkwoodsche Korrelationsfaktoren von binaren Systemen mit Alkoholen und Diethylether. J. Prakt. Chem. 1977, 319, 353-361.

(27) Sharma, V.; Thakur, N.; Sharma, D. R.; Negi, N. S.; Rangra, V. S. Dielectric Relaxation Study of Ethanol in Benzene from Microwave Absorption Data. Z. Naturforsch., A: Phys. Sci. 2007, 62, 406-408.

(28) Ball, P. Water as an Active Constituent in Cell Biology. Chem. Rev. 2008, 108, 74-108.

(29) Paoli, A.; Schiraldi, A. Developments in Food Science. In Food Flavors: Generation, Analysis and Process Influence Proceedings of the 8th International Flavor Conference; Charalambous, G., Ed.; Elsevier, 1995; Vol. 37; pp 1065-1072.

(30) Feng, Q. L.; Ning, P.; Wang, X. Q.; Jia, Y. Study on Microwave Desorption Azeotropic Distillation of Ethanol-Loaded Activated Carbon under Vacuum Condition. Appl. Mech. Mater. 2013, 295298, 1240-1244.

(31) Komorowska-Durka, M.; van Houten, R.; Stefanidis, G. D. Application of Microwave Heating to Pervaporation: A Case Study for Separation of Ethanol-Water Mixtures. Chem. Eng. Process. 2014, 81, $35-40$.

(32) Olmi, R.; Meriakri, V. V.; Ignesti, A.; Priori, S.; Riminesi, C. Monitoring Alcoholic Fermentation by Microwave Dielectric Spectroscopy. J. Microwave Power 2007, 41, 38-49.

(33) Li, R.; D’Agostino, C.; McGregor, J.; Mantle, M. D.; Zeitler, J. A.; Gladden, L. F. Mesoscopic Structuring and Dynamics of Alcohol/ Water Solutions Probed by Terahertz Time-Domain Spectroscopy and Pulsed Field Gradient Nuclear Magnetic Resonance. J. Phys. Chem. B 2014, 118, 10156-10166.

(34) Petong, P.; Pottel, R.; Kaatze, U. Dielectric Relaxation of HBonded Liquids. Mixtures of Ethanol and n-Hexanol at Different Compositions and Temperatures. J. Phys. Chem. A 1999, 103, 61146121.

(35) Jorgensen, W. L. Optimized Intermolecular Potential Functions for Liquid Alcohols. J. Phys. Chem. 1986, 90, 1276-1284.

(36) Saiz, L.; Padró, J. A.; Guàrdia, E. Structure and Dynamics of Liquid Ethanol. J. Phys. Chem. B 1997, 101, 78-86.

(37) Frank, H. S.; Evans, M. W. Free Volume and Entropy in Condensed Systems III. Entropy in Binary Liquid Mixtures; Partial Molal Entropy in Dilute Solutions; Structure and Thermodynamics in Aqueous Electrolytes. J. Chem. Phys. 1945, 13, 507-532.

(38) Dixit, S.; Soper, A. K.; Finney, J. L.; Crain, J. Water Structure and Solute Association in Dilute Aqueous Methanol. Europhys. Lett. 2002, 59, 377-383.

(39) Raschke, T. M.; Levitt, M. Nonpolar Solutes Enhance Water Structure Within Hydration Shells while Reducing Interactions between Them. Proc. Natl. Acad. Sci. U. S. A. 2005, 102, 6777-6782.

(40) Laage, D.; Stirnemann, G.; Hynes, J. T. Why Water Reorientation Slows without Iceberg Formation around Hydrophobic Solutes. J. Phys. Chem. B 2009, 113, 2428-2435.
(41) Petersen, C.; Tielrooij, K.; Bakker, H. J. Strong Temperature Dependence of Water Reorientation in Hydrophobic Hydration Shells. J. Chem. Phys. 2009, 130, 214511.

(42) Garde, S.; Patel, A. J. Unraveling the Hydrophobic Effect, One Molecule at a Time. Proc. Natl. Acad. Sci. U. S. A. 2011, 108, 1649116492.

(43) Guevara-Carrion, G.; Vrabec, J.; Hasse, H. Prediction of Selfdiffusion Coefficient and Shear viscosity of Water and its Binary Mixtures with Methanol and Ethanol by Molecular Simulation. J. Chem. Phys. 2011, 134, 074508.

(44) Gereben, O.; Pusztai, L. Investigation of the Structure of Ethanol-Water Mixtures by Molecular Dynamics Simulation I: Analyses Concerning the Hydrogen-Bonded Pairs. J. Phys. Chem. B 2015, 119, 3070-3084.

(45) Noskov, S. Y.; Lamoureux, G.; Roux, B. Molecular Dynamics Study of Hydration in Ethanol-Water Mixtures Using a Polarizable Force Field. J. Phys. Chem. B 2005, 109, 6705-6713.

(46) Zhong, Y.; Patel, S. Electrostatic Polarization Effects and Hydrophobic Hydration in Ethanol-Water Solutions from Molecular Dynamics Simulations. J. Phys. Chem. B 2009, 113, 767-778.

(47) Davis, J. G.; Gierszal, K. P.; Wang, P.; Ben-Amotz, D. Water Structural Transformation at Molecular Hydrophobic Interfaces. Nature 2012, 491, 582-585.

(48) Galamba, N. Water's Structure around Hydrophobic Solutes and the Iceberg Model. J. Phys. Chem. B 2013, 117, 2153-2159.

(49) Graziano, G. Comment on 'Water's Structure around Hydrophobic Solutes and the Iceberg Model. J. Phys. Chem. B 2014, $118,2598-2599$.

(50) Galamba, N. Reply to 'Comment on 'Water's Structure around Hydrophobic Solutes and the Iceberg Model'. J. Phys. Chem. B 2014, $118,2600-2603$.

(51) Ghoufi, A.; Artzner, F.; Malfreyt, P. Physical Properties and Hydrogen-Bonding Network of Water-Ethanol Mixtures from Molecular Dynamics Simulations. J. Phys. Chem. B 2016, 120, 793802.

(52) Lou, J.; Hatton, T. A.; Laibinis, P. E. Effective Dielectric Properties of Solvent Mixtures at Microwave Frequencies. J. Phys. Chem. A 1997, 101, 5262-5268.

(53) Sihvola, A. Mixing Rules with Complex Dielectric Coefficients. Subsurface Sensing Technologies and Applications 2000, 1, 393-415.

(54) Kremer, F.; Schönhals, A.; Lunkenheimer, P.; Loidl, A.; Huwe, A.; Rozanski, S. A.; Floudas, G.; Mijovic, J.; Hartmann, L.; Fukao, K. et al. In Broadband Dielectric Spectroscopy; Kremer, F., Schönhals, A., Eds.; Springer, 2003.

(55) Carpi, F., De Rossi, D., Kornbluh, R., Pelrine, R., SommerLarsen, P., Eds. Dielectric Elastomers as Electromechanical Transducers: Fundamentals, Materials, Devices, Models and Applications of an Emerging Electroactive Polymer Technology; Elsevier Science, 2008.

(56) Álvarez, A.; Fayos-Fernández, J.; Monzó-Cabrera, J.; Cocero, M. J.; Mato, R. B. Measurement and Correlation of the Dielectric Properties of a Grape Pomace Extraction Media. Effect of Temperature and Composition. J. Food Eng. 2017, 197, 98-106.

(57) Zasetsky, A. Y.; Lileev, A. S.; Lyashchenko, A. K. Molecular Dynamic Simulations of Terahertz Spectra for Water-Methanol Mixtures. Mol. Phys. 2010, 108, 649-656.

(58) Olmi, R; Bittelli, M. Can Molecular Dynamics Help in Understanding Dielectric Phenomena? Meas. Sci. Technol. 2017, 28, 014003.

(59) Cardona, J.; Fartaria, R.; Sweatman, M. B.; Lue, L. Molecular Dynamics Simulations for the Prediction of the Dielectric Spectra of Alcohols, Glycols and Monoethanolamine. Mol. Simul. 2016, 42, 370390.

(60) Chen, B.; Potoff, J. J.; Siepmann, J. I. Monte Carlo Calculations for Alcohols and Their Mixtures with Alkanes. Transferable Potentials for Phase Equilibria. 5. United-Atom Description of Primary, Secondary, and Tertiary Alcohols. J. Phys. Chem. B 2001, 105, 3093-3104. 
(61) Stubbs, J. M.; Potoff, J. J.; Siepmann, J. I. Transferable Potentials for Phase Equilibria. 6. United-Atom Description for Ethers, Glycols, Ketones, and Aldehydes. J. Phys. Chem. B 2004, 108, 17596-17605.

(62) Wu, Y.; Tepper, H. L.; Voth, G. A. Flexible Simple Point-charge Water Model with Improved Liquid-state Properties. J. Chem. Phys. 2006, 124, 024503.

(63) Hess, B.; Kutzner, C.; van der Spoel, D.; Lindahl, E. GROMACS 4: Algorithms for Highly Efficient, Load-Balanced, and Scalable Molecular Simulation. J. Chem. Theory Comput. 2008, 4, 435-447.

(64) Hockney, R. W.; Goel, S. P.; Eastwood, J. W. Quiet Highresolution Computer Models of a Plasma. J. Comput. Phys. 1974, 14, $148-158$.

(65) Nosé, S. A Molecular Dynamics Method for Simulations in the Canonical Ensemble. Mol. Phys. 1984, 52, 255-268.

(66) Hoover, W. G. Canonical Dynamics: Equilibrium Phase-space Distributions. Phys. Rev. A: At., Mol., Opt. Phys. 1985, 31, 1695-1697.

(67) Parrinello, M.; Rahman, A. Polymorphic Transitions in Single Crystals: A New Molecular Dynamics Method. J. Appl. Phys. 1981, 52, 7182-7190.

(68) Nosé, S.; Klein, M. Constant Pressure Molecular Dynamics for Molecular Systems. Mol. Phys. 1983, 50, 1055-1076.

(69) Hess, B.; Bekker, H.; Berendsen, H. J. C.; Fraaije, J. G. E. M. LINCS: A Linear Constraint Solver for Molecular Simulations. J. Comput. Chem. 1997, 18, 1463-1472.

(70) Darden, T.; York, D.; Pedersen, L. Particle Mesh Ewald: An N. $\log (\mathrm{N})$ Method for Ewald Sums in Large Systems. J. Chem. Phys. 1993, 98, 10089-10092.

(71) de Leeuw, S. W.; Perram, J. W.; Smith, E. R. Simulation of Electrostatic Systems in Periodic Boundary Conditions. I. Lattice Sums and Dielectric Constants. Proc. R. Soc. London, Ser. A 1980, 373, 2756.

(72) Neumann, M.; Steinhauser, O. On the Calculation of the Frequency-dependent Dielectric Constant in Computer Simulations. Chem. Phys. Lett. 1983, 102, 508-513.

(73) Powell, M. J. D. The BOBYQA Algorithm for Bound Constrained Optimization Without Derivatives. Department of Applied Mathematics and Theoretical Physics, Cambridge University, 2009.

(74) Gereben, O.; Pusztai, L. On the Accurate Calculation of the Dielectric Constant from Molecular Dynamics Simulations: The case of SPC/E and SWM4-DP Water. Chem. Phys. Lett. 2011, 507, 80-83.

(75) Raabe, G.; Sadus, R. J. Molecular Dynamics Simulation of the Dielectric Constant of Water: The Effect of Bond Flexibility. J. Chem. Phys. 2011, 134, 234501.

(76) Caleman, C.; van Maaren, P. J.; Hong, M.; Hub, J. S.; Costa, L. T.; van der Spoel, D. Force Field Benchmark of Organic Liquids: Density, Enthalpy of Vaporization, Heat Capacities, Surface Tension, Isothermal Compressibility, Volumetric Expansion Coefficient, and Dielectric Constant. J. Chem. Theory Comput. 2012, 8, 61-74.

(77) Tanaka, H.; Ohmine, I. Large Local Energy Fluctuations in Water. J. Chem. Phys. 1987, 87, 6128-6139.

(78) Ohmine, I.; Tanaka, H.; Wolynes, P. G. Large Local Energy Fluctuations in Water. II. Cooperative Motions and Fluctuations. J. Chem. Phys. 1988, 89, 5852-5860.

(79) Barthel, J.; Bachhuber, K.; Buchner, R.; Hetzenauer, H. Dielectric Spectra of Some Common Solvents in the Microwave Region. Water and Lower Alcohols. Chem. Phys. Lett. 1990, 165, 369373.

(80) Rønne, C.; Thrane, L.; Åstrand, P.-O.; Wallqvist, A.; Mikkelsen, K. V.; Keiding, S. R. Investigation of the Temperature Dependence of Dielectric Relaxation in Liquid Water by $\mathrm{THz}$ Reflection Spectroscopy and Molecular Dynamics Simulation. J. Chem. Phys. 1997, 107, 53195331.

(81) Starr, F. W.; Nielsen, J. K.; Stanley, H. E. Fast and Slow Dynamics of Hydrogen Bonds in Liquid Water. Phys. Rev. Lett. 1999, 82, 2294-2297. 